\title{
LA GESTIÓN DE CONTENIDOS COMO ACTIVIDAD ESTRATÉGICA EN EMPRESAS DE RADIODIFUSIÓN. ESTUDIO DE CASOS EN LA RADIO COMERCIAL ESPAÑOLA
}

\author{
Manuel Fernández-Sande, Dolores Rodríguez-Barba y Miriam Rodríguez- \\ Pallares
}
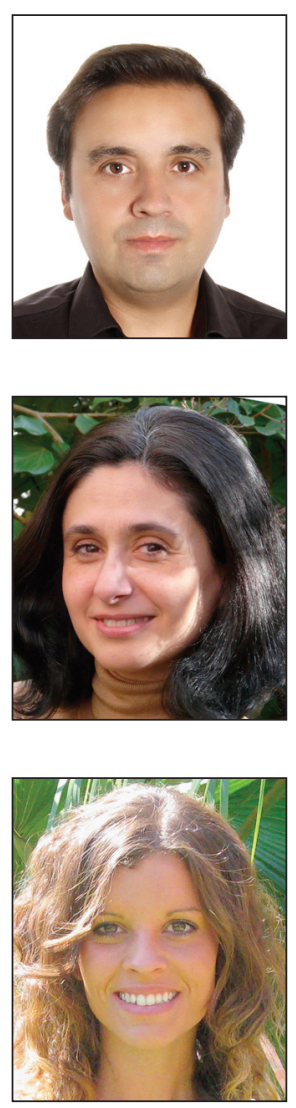

Manuel Fernández-Sande, doctor en ciencias de la información por la Univ. Complutense de Madrid (UCM), licenciado en periodismo (UCM) y en documentación (Univ. Carlos III de Madrid), es profesor en la Fac. de Ciencias de la Información (UCM). Investiga sobre el mercado radiofónico europeo, historia de la radio, dirección estratégica de empresas de comunicación, y organización y gestión del conocimiento. Es autor de Los orígenes de la radio en España I y II (2006) y codirector de Panorámica de la comunicación y de los medios en Brasil y España (2013).

http://orcid.org/0000-0002-0740-2630

manuel.fernandez@ucm.es

Dolores Rodríguez-Barba es doctora en ciencias de la información. Trabaja en el Departamento de Periodismo IV de la Universidad Complutense de Madrid como profesora en el Grado y Master oficial de Periodismo, y como investigadora en el ámbito de la organización y gestión de empresas de comunicación. Ha trabajado en TVE y en las áreas de programación y dirección técnica en varias cadenas de radio. Es miembro del grupo de investigación MediaCom UCM y co-fundadora de Ocendi.

http://orcid.org/0000-0001-8437-5536

lolar@ucm.es

Miriam Rodríguez-Pallares es diplomada en biblioteconomía y documentación por la Univ. de Salamanca, máster en periodismo por la UCM y doctoranda en el Depto. de Periodismo IV de la UCM. Su trabajo fin de máster lleva por título: La documentación informativa en los medios de comunicación. Perspectiva económica y funcional. Desde 2007 ha trabajado en el área de documentación y gestión de contenidos de El país, en el Área de Gobierno de Familia y Servicios Sociales del Ayuntamiento de Madrid y en Everis BPO; como periodista, en la Unión de Asociaciones de Estanqueros de España y como colaboradora de la Fundación Mapfre.

http://orcid.org/0000-0002-5486-0298

mrpallares@pdi.ucm.es Universidad Complutense de Madrid Facultad de Ciencias de la Información, Departamento de Periodismo IV Avda. Complutense s/n. 28040 Madrid, España

\section{Resumen}

Se presentan los resultados parciales del análisis comparativo realizado en tres de las principales cadenas de radiodifusión comercial españolas (Cadena Ser, Onda Cero y Cope) para identificar y analizar la estructura de los departamentos encargados de la gestión de contenidos así como el modelo y las herramientas de gestión. El estudio se presenta aplicando la metodología de casos y desde una perspectiva funcional, es decir, en relación con la actividad principal de cada una de las empresas, que fundamenta su modelo de negocio en la comercialización de contenidos informativos, culturales y de entretenimiento. En la segunda fase del análisis, se han realizado entrevistas en profundidad a los responsables de los cuadros altos de los organigramas de las empresas radiofónicas.

\section{Palabras clave}

Sistemas de gestión de contenidos, SGC, Empresas de radiodifusión, Documentación en medios, Documentación radiofónica, Cadena Ser, Onda Cero, Cope, Enciclomedia, Dalet Plus, Paloo.

Title: Content management as a strategic activity in broadcasting companies. Case studies about private radio networks in Spain 


\begin{abstract}
We present partial results of a comparative analysis in three major commercial broadcasters in Spain -Cadena Ser, Onda Cero and Cope- to identify and analyze the structure of the departments responsible for content management, as well as the model and management tools used. The study applies a case methodology and takes a functional perspective, that is, a business model based on the core business of each company, i.e., informational, cultural, or entertainment content. For the second phase of the analysis, we conducted in-depth interviews with the heads of the executive team of each broadcasting company.
\end{abstract}

\title{
Keywords
}

Content management systems, CMS, Broadcasting companies, Media documentation, Broadcasting documentation, Spain, Cadena Ser, Onda Cero, Cope, Enciclomedia, Dalet Plus, Paloo.

Fernández-Sande, Manuel; Rodríguez-Barba, Dolores; Rodríguez-Pallares, Miriam (2013). "La gestión de contenidos como actividad estratégica en empresas de radiodifusión. Estudio de casos en la radio comercial española". El profesional de la información, septiembre-octubre, v. 22, n. 5, pp. 392-398

http://dx.doi.org/10.3145/epi.2013.sep.03

\section{Introducción}

Las organizaciones empresariales llevan a cabo políticas estratégicas, con objetivos de superación y competitividad, que complementan los factores productivos propios de la economía industrial -capital, trabajo y recursos humanos- y se basan en la innovación tecnológica y organizativa como factores de éxito. Esto supone una constante vigilancia crítica de la actividad empresarial aun en épocas de estabilidad: "la cultura de lo intangible está dejando paso a una nueva manera de generar riqueza" (Carrillo-Durán; Nuño-Moral, 2010).

En este contexto la gestión de contenidos en la industria de la comunicación cobra un doble protagonismo (RodríguezPallares, 2012):

- desde una perspectiva funcional, enfocada a la mejora de la distribución y la revalorización de su producción y, por lo tanto de su marca en el mercado, que busca informar, formar y entretener;

- desde una perspectiva estratégica, enfocada a la obtención de beneficios a largo plazo, que pueden repercutir en el aprendizaje organizacional.

La empresa radiofónica ha experimentado en los últimos años una importante evolución en paralelo a los procesos de transformación de los mercados informativos. Podemos definirla como una "organización social que, mediante la gestión y administración de elementos humanos, técnicos, tangibles, intangibles y recursos financieros, produce, distribuye -por medio de diferentes soportes y canales- y comercializa contenidos sonoros y otros recursos multimedia que pretenden satisfacer las necesidades informativas $y$ de entretenimiento de sus públicos objetivos, con el fin de obtener el máximo beneficio posible". (Fernández-Sande; Peinado-Miguel, 2012).

Estas empresas han incorporado la gestión de contenidos sonoros y multimedia como un activo fundamental de sus sistemas productivos y de comercialización. La gestión de contenidos es un proceso complejo que requiere políticas de gestión y un software especializado, que denominamos sistemas gestores de contenido (content management sys- tems o CMS) (Cox; Yeadon, 2002). Implica un conjunto de destrezas y actuaciones profesionales que permiten un tratamiento sistemático y global de los diferentes tipos de información (García-Morales; Bustelo-Ruesta, 2001). En el concepto de gestión de contenidos confluyen por tanto componentes tecnológicos, estrategia empresarial y prácticas profesionales. El objetivo final de todo proceso de gestión de contenidos es la creación, gestión, presentación y difusión de información (Pérez-Montoro, 2005) con la mayor eficiencia y eficacia posible. Para lograrlo se requiere un único centro documental que disponga de una plataforma tecnológica capaz de ofrecer servicio a todos los editores y productores que trabajen en red en la empresa radiofónica (García-Lastra, 2012).

\section{En el concepto de gestión de contenidos confluyen componentes tecnológicos, estrategia empresarial y prácticas profe- sionales}

La bibliografía científica ha tratado de establecer una diferenciación conceptual entre gestión del conocimiento y gestión de contenidos. Delimitar ambos procesos resulta necesario desde un punto de vista teórico pero también es importante para el correcto funcionamiento organizacional. La interacción permanente entre la generación de conocimiento y la gestión de contenidos dificulta en la práctica esta delimitación. La primera se centra en el diseño de estrategias, procesos y estructuras que permitan a la organización incorporar y aprovechar el conocimiento de todos sus miembros (Fernández-Marcial, 2006). La mayoría de autores coinciden en que se encuentra en un plano superior a la gestión de contenidos aunque ambos procesos son totalmente interdependientes. (Nonaka; Takeuchi, 1995; Newman, 1997; Pearson, 1999; Davenport; Prusak, 2001; Bueno-Campos, 2004)

\section{Objetivos y metodología de la investigación}

El objetivo general es conocer los modelos de gestión de contenidos desde una perspectiva funcional de tres de las 
principales cadenas de radiodifusión privadas de España: Ser, Onda Cero y Cope. En concreto, se pretende:

- identificar y analizar la estructura de los departamentos encargados de la gestión de contenidos;

- identificar la visibilidad de la gestión de contenidos en el seno de la organización;

- identificar el modelo y las herramientas de gestión de contenidos.

La metodología empleada es la del estudio de casos en tres fases:

- análisis exhaustivo de la bibliografía científica y profesional;

- recogida de documentación propia de cada una de las empresas;

- entrevistas en profundidad a los responsables de los cuadros directivos de cada medio, realizadas entre julio de 2010 y abril de 2013 (la relación de entrevistados se muestra al final del artículo).

\section{Modelos de gestión de contenidos en Cadena Ser, Onda Cero y Cope}

\subsection{Identificación del departamento responsable}

No existen dudas sobre la importancia de la gestión de contenidos en el diseño general de estrategias empresariales, especialmente en las empresas de comunicación, que deben dar prioridad a la organización de la producción y la explotación de dichos contenidos atendiendo a criterios documentales con un fin informativo y de difusión y no sólo probatorio. Sin embargo, en los últimos años, a consecuencia de los procesos de convergencia tecnológica se producen situaciones dispares en el conjunto de los medios, motivadas por estrategias no muy definidas: creación de líneas de negocio cuyos objetivos se cumplen en el primer año y que tras superar el período de viabilidad son suprimidas por falta de rentabilidad (Peinado-Miguel; Rodríguez-Barba, 2012), simples logros a exhibir, o actividades residuales.

En los casos estudiados, Cadena Ser es la única empresa radiofónica con un departamento de documentación corporativa que desempeña todas las funciones propias de la gestión de contenidos con fin informativo, incluyendo producción y publicación. Cope por su parte dispone de un departamento de documentación orientado hacia la preservación de contenidos. Por último Onda Cero carece en su estructura organizativa de un servicio especializado a pesar de contar con un CMS que mantiene los contenidos durante 1 año en su división analógica (tabla 1).

Tabla 1: Modelos de gestión de contenidos

\begin{tabular}{|c|c|l|c|}
\hline Empresa & \multicolumn{1}{|c|}{ CMS } & \multicolumn{1}{|c|}{ Uso } & Alcance \\
\hline Ser & Enciclomedia & $\begin{array}{l}\text { Descripción y gestión } \\
\text { Repositorio } \\
\text { Plataforma de publicación }\end{array}$ & Prisa Radio \\
\hline $\begin{array}{c}\text { Onda } \\
\text { Cero }\end{array}$ & Dalet & $\begin{array}{l}\text { Repositorio temporal (1 año) } \\
\text { Acceso a fondos de A3TV }\end{array}$ & $\begin{array}{l}\text { Onda Cero } \\
\text { Europa FM }\end{array}$ \\
\hline Cope & Knosys & $\begin{array}{c}\text { Cope } \\
\text { Descripción y gestión }\end{array}$ & $\begin{array}{c}\text { Cadena 100 } \\
\text { Rock FM }\end{array}$ \\
\hline
\end{tabular}

\subsection{Posicionamiento organizativo y evolución de la gestión de contenidos}

El proceso de implantación del modelo de gestión de contenidos en un medio, las razones que han llevado a tal decisión y la evolución posterior proporciona las claves para conocer su funcionalidad y valoración empresarial, su peso en la organización.

Es imposible impermeabilizar los modelos de gestión de contenidos vigentes en las emisoras analizadas de la influencia que, tanto a nivel funcional como organizativo, ejercen sobre ellas sus empresas matrices. El modelo de gestión de contenidos de la Cadena Ser se entiende teniendo en cuenta que es la cabecera de la red internacional que conforma Prisa Radio; el de Onda Cero en el marco de Atresmedia. Los modelos organizativos divisionales ya sean geográficos o por productos (De-Mateo; Bergés; Sabater, 2009) determinan el diseño y el posicionamiento organizacional de la gestión de contenidos en la empresa.

\section{Cadena Ser es la única empresa con un departamento de documentación que realiza todas las funciones propias de la gestión de contenidos con fin infor- mativo}

El Departamento de Documentación de la Cadena Ser fue creado en 1988, tras la inclusión de la emisora en el accionariado del Grupo Prisa, con el fin de salvaguardar la documentación sonora con la que contaba la marca radiofónica más longeva de España. Su labor de archivo no fue cuestión baladí por la novedad que éste suponía en el sector privado. El cambio de paradigma en cuanto a gestión de contenidos llegó a Prisa Radio (entonces Unión Radio) en 2004. El objetivo era pasar de un modelo local a un modelo integracionista que vinculase el total del valor informativo generado en todas las sedes de Prisa Radio en un mismo repositorio de datos que nutriese, a su vez, a todo el conjunto de emisoras del grupo a ambos lados del Atlántico. Se optó por un programa gestor de contenidos propio, denominado Enciclomedia, que empezó a desarrollar en 2001 la empresa Atos Consulting. Paralelamente se fue diseñando y organizando el Departamento de Documentación con un concepto más funcional, hasta llegar a denominarse primero Gestión de Contenidos y en la actualidad Documentación Corporativa.

En 2012 Documentación Corporativa amplió sus competencias para asumir procesos editoriales secundarios, es decir, para convertirse en publicador de contenidos y no sólo en descriptor y gestor. Para ello, se reforzó la vinculación del programa de gestión de contenidos con las líneas de negocio online pese a que, como en todos los casos analizados, siguen siendo líneas de actuación independientes orgánica y funcionalmente. Enciclomedia dispone de pasarelas automáticas que la convierten en una plataforma de lanzamiento directo hacia publicaciones webs, telefonía móvil, apps, etc. Estas funciones representan un importante salto cualitativo respecto a las bases de datos "estáticas". 
La aportación documental a la programación de la Cadena Ser es la más visible de los 3 casos analizados:

- por el apoyo al trabajo de las redacciones en sus labores retrospectivas y comparativas;

- por la valoración del trabajo documental en el medio, hecho que distingue este caso de todos los demás y denota un reconocimiento empresarial a esta labor que repercute directamente en el producto y en la organización, particularmente en la plantilla.

\section{Atresmedia Radio justifica la ausencia de un departamento propio en Onda Cero alegando la posibilidad de acceso al re- positorio Atresmedia TV, sin plantearse un repositorio conjunto}

El caso de Onda Cero es diferente. No existe un departamento de gestión de contenidos que colabore con la producción informativa y en el proceso de reutilización y rentabilización de los contenidos generados por el grupo Atresmedia Radio. Onda Cero cuenta tan sólo con un archivo físico donde se almacena el continuo de su programación on air (en vivo, no enlatada), con códigos descriptivos temporales, lo que significa que la búsqueda y reutilización de sus contenidos propios depende de un responsable del archivo, los contenidos no están vinculados y el usuario debe conocer la franja horaria en la que ha sido emitido su objeto de búsqueda. Atresmedia Radio justifica la ausencia de un departamento propio a nivel funcional alegando que Atresmedia TV da acceso a Onda Cero al repositorio de datos documentales de la televisión -gestionado externamente por Accenture-, solventando sus necesidades informativas más inmediatas. También se apunta el ahorro de costes para el grupo mediático, aun cuando parece que la rentabilización real vendría de la mano de un modelo de gestión y archivo común para todos los contenidos.
Cope por su parte cuenta con un departamento de gestión de contenidos, Documentación, creado en 1989 tras reconfiguraciones empresariales internas. Se creó con el propósito de no perder horas de grabación de incalculable valor, parte de las cuales salieron por las puertas de Radio Popular sin más explicación que la marcha de sus locutores estrella y un antiguo modelo de archivo de fichas arcaico, poco exhaustivo y de escasa supervisión. Hasta ese momento, alentada por su naturaleza de "radio de estrellas", la gestión y custodia de los contenidos de audio en la Cope era competencia de cada programa. Las copias legales estaban sujetas a una normativa hoy ya caduca que exigía la conservación durante sólo un mes de la emisión en antena en soporte cassette, frente a los tres meses que obliga la ley actual. Desde su creación, el Departamento de Documentación ha sido objeto de pocos cambios y cuenta con recursos limitados tanto informáticos como humanos (actualmente dos personas).

En los casos analizados se han encontrado tres ejemplos diferentes de posicionamiento organizativo del área de gestión de contenidos:

- en la Cadena Ser el Departamento de Documentación Corporativa depende de la presidencia de Prisa Radio;

- en la Cope depende de la dirección de programas;

- en Onda Cero no existe unidad departamental de gestión de contenidos.

En los tres casos la ubicación del departamento de documentación en la estructura de la empresa permite deducir el nivel de reconocimiento y el potencial, tanto organizativo como funcional, del que dispone la gestión de contenidos.

\subsection{Modelos y aplicaciones de gestión de contenidos}

Los sistemas de gestión de contenidos pueden dividirse en WCM (web content management) y ECM (enterprise content management). Ambos abarcan tareas de creación, gestión, distribución, y publicación de la información en un modelo centralizado (Robertson, 2003). Cada organización

Tabla 2: Departamentos y herramientas de gestión de contenidos Cadena Ser, Onda Cero y Cope.

\begin{tabular}{|c|c|c|c|}
\hline \multicolumn{4}{|c|}{ Servicio documental y actual gestor de contenidos } \\
\hline $\begin{array}{l}\text { Fecha de creación del departa- } \\
\text { mento de gestión de contenidos }\end{array}$ & 1988 & No existe & 1989 \\
\hline CMS/MAM & Sí, permanente (Enciclomedia) & $\begin{array}{c}\text { Sí, conservación temporal y con } \\
\text { prestaciones limitadas } \\
\text { (Dalet Plus) }\end{array}$ & $\begin{array}{l}\text { Sí, permanente (Knosys) + WCM } \\
\text { independiente (Paloo) }\end{array}$ \\
\hline $\begin{array}{l}\text { Naturaleza del programa actual } \\
\text { (adquisición/propia) }\end{array}$ & Propia & Adquisición & Adquisición \\
\hline \multicolumn{4}{|c|}{ Organigrama departamental } \\
\hline Dependencia del departamento & Dirección General de Prisa Radio & $\begin{array}{c}\text { Fonoteca depende del Director de } \\
\text { Emisiones y Subdirector de Progra- } \\
\text { mas }\end{array}$ & Dirección de Programas \\
\hline $\begin{array}{l}\text { Cabecera del servicio de gestión } \\
\text { de contenidos }\end{array}$ & Sí & No & Sí \\
\hline Ubicación del departamento & $\begin{array}{l}\text { Sede principal de Prisa Radio. } \\
\text { Gran Vía, 32. } 28013 \text { Madrid }\end{array}$ & No hay departamento & $\begin{array}{c}\text { Sede principal Cope. } \\
\text { Alfonso XI, 4. } 28014 \text { Madrid }\end{array}$ \\
\hline
\end{tabular}


adapta el CMS a sus necesidades particulares. Así, en las empresas de comunicación se usa comúnmente la nomenclatura MAM (media asset management) para identificar un CMS que, manteniendo las mismas bases conceptuales, no se basa en un modelo estándar, independientemente de si se trata de un gestor web o no. Lo más habitual es que se adapten productos prefabricados a las exigencias del contexto de aplicación -Onda Cero con el MAM de Dalet-, o que sea la empresa quien diseñe su propia aplicación -Cadena Ser con Enciclomedia-.

Radio Popular cuenta con un departamento de gestión de contenidos con limitaciones funcionales que pierde peso organizativo frente a la división digital

El modelo de gestión de contenidos de Prisa Radio está condicionado fundamentalmente por su naturaleza internacional. El grupo radiofónico cuenta con dos grandes sistemas de actividad en su modelo:

- Local, basado en la aplicación Redacta: cada emisora cuenta con una aplicación identificada como un entorno de edición y archivo local para los periodistas, con campos de descripción reducidos y para uso interno. Los sistemas locales de las diferentes emisoras están interconectados entre sí para facilitar el intercambio de archivos.

- En cadena, basado en la aplicación Enciclomedia: cuando el contenido manejado en una emisora local concreta traspasa los intereses locales para alcanzar transcendencia para toda la marca, el contenido es analizado y seleccionado para pasar a formar parte del CMS.

La internacionalidad de su modelo de gestión de contenidos exige una descripción de perfiles de usuario muy definida con el fin de evitar duplicaciones y accesos dobles a la manipulación de contenidos. Por esta razón, se ha diseñado un modelo jerarquizado cuya cabecera está en Madrid y de la cual dependen orgánicamente los demás puntos clave con permiso para nutrir el sistema en cadena: España, Colombia, Chile, Argentina y México.

Por su parte, el contexto de trabajo de Onda Cero Radio se divide en tres grandes áreas:

- entorno de producción: cuyo soporte informático es el gestor de contenidos Dalet, donde se custodian los continuos durante un período máximo de un año, con una ficha descriptiva generada por los propios periodistas; pasado este tiempo, pasan al departamento de Fonoteca en formato físico, que cuenta con una base de datos simple de acceso únicamente para el departamento;

- entorno de emisión: cuyo soporte informático es a día de hoy Viva NT;

- entorno de intercambio de contenidos entre las diferentes emisoras de Onda Cero por FTP.

Resulta desconcertante que Onda Cero haya hecho una importante inversión en la adquisición de un MAM, pero no rentabilice las prestaciones de un gestor de contenidos como fuente, sino tan sólo como salvaguarda temporal de su emisión on air. Evidentemente, la custodia de sus propios fondos garantiza la posibilidad de recuperarlos pese al consiguiente retraso que implica su archivo físico, pero en ningún caso multiplica su valor ni favorece la rentabilización del trabajo realizado. Podría decirse que se mantiene un modelo de custodia permanente de copias de seguridad. La ausencia de una actividad de descripción y vinculación de contenidos exhaustiva evita la diferenciación de perfiles de usuario, incluso en la Fonoteca, donde los dos responsables, aun teniendo vinculación jerárquica, intercalan privilegios de actuación.

Por último, la actividad documental de Cope en el área no digital basa su objetivo primario en la custodia de los continuos y todo corte de audio preseleccionado por los redactores y editores de programas e informativos y extraído de su propio sistema de emisión. En ocasiones puntuales funciona como fuente y genera contenidos documentales coadyuvantes para la producción informativa. El modelo de gestión de contenidos se sustenta en el gestor documental Knosys y las prestaciones del paquete Office de Microsoft. Una plantilla de tan sólo dos profesionales vinculados jerárquicamente exime la necesidad de definir perfiles de usuario, compartiendo ambos los privilegios de actuación.

Las empresas radiofónicas comerciales en España carecen de un planteamiento estratégico global que les permita integrar el potencial de la gestión de contenidos en la actividad corporativa

Actualmente se está estudiando la posibilidad de unificar todo el proceso de producción radiofónica en un único CMS, desde los sistemas de producción, emisiones y archivo, al multimedia. De llevarse a cabo este proyecto, podría posicionar al Grupo Radio Popular en la vanguardia en gestión de contenidos en el panorama nacional. El objetivo es la vinculación total de los contenidos de la empresa en un mismo sistema integrado sustentado en una compleja arquitectura informática basada en capas semánticas inteligentes. El salto cualitativo sería revolucionario para Radio Popular (Luis Martín-Pérez, 9 de abril de 2013).

\section{Conclusiones}

Las tres empresas radiofónicas analizadas cuentan con modelos de gestión de contenidos que presentan grandes diferencias, tanto en sus aspectos organizativos como funcionales. En ningún caso se identifica un planteamiento estratégico global que permita integrar y aprovechar el potencial de la gestión de contenidos en la actividad corporativa. Se mantiene todavía muy arraigada la concepción del archivo sonoro estático y se detecta una infraexplotación de los contenidos sonoros y multimedia a pesar del importante desarrollo en lo que se refiere a herramientas tecnológicas y prácticas profesionales registradas en los últimos años.

De los tres casos analizados Prisa Radio representa el modelo de gestión de contenidos más evolucionado. Dispone de un departamento de documentación consolidado en su 
estructura organizativa, capaz de desarrollar procesos de producción propia y difusión de información aportando de esa forma un valor añadido más visible a la oferta radiofónica. También se diferencia por haber desarrollado un CMS de creación propia adaptado a sus características y objetivos, Enciclomedia, que posibilita un modelo de trabajo en red internacional.

Prisa Radio ha consolidado la gestión de contenidos como parte importante de su cultura y estrategia empresarial, a pesar de la inestabilidad que provocan los cambios organizativos que se han producido y que se puedan producir en un futuro, todo indica que en los próximos años se intensificará la rentabilización de los contenidos y se reforzará el área de Documentación Corporativa.

Se detecta una infraexplotación de los contenidos sonoros y multimedia

Las otras dos empresas analizadas, han optado o bien por la externalización de los procesos de gestión, caso de Atresmedia y Accenture; o por la adquisición de aplicaciones informáticas externas que requieren una posterior adaptación, caso de Cope con Knosys y Paloo.

En Onda Cero no parece que la empresa matriz decida a corto plazo crear un departamento de gestión de contenidos propio con capacidad de generar valor añadido. Por su parte Cope se plantea mejoras cualitativas en su modelo de gestión de contenidos, pero este posible desarrollo tecnológico, funcional y estratégico parece condicionado por la evolución económica del grupo y su capacidad de generar nuevas vías de financiación.

Por último hay que destacar que los perfiles de usuario entre los gestores de contenido son más rígidos cuanto más grande y jerarquizada es la red de trabajo, y más polivalentes cuanto más pequeña y menos definida es la misma.

\section{Entrevistas}

Ángeles Afuera (abril 2012)

Jefa del Departamento de Documentación Corporativa de la Cadena Ser.

Miguel-Ángel Álbez-Sánchez (junio 2012)

Responsable de la Fonoteca y Archivo Sonoro de Onda Cero.

Francisco Castaño-Igual (junio 2012)

Jefe del Departamento de Informática y Sistemas de Onda cero.

Ester Turu-Llimós (junio 2012)

Subdirectora de Informativos de Onda Cero.

Alicia Sánchez-Gómez (julio 2010)

Documentalista de la Cadena Ser Madrid.

Luis Rodríguez-Pi (agosto 2012)

Director de Antena de la Cadena Ser Madrid.

Alicia Ayala (agosto 2012)

Documentalista de la Cadena Cope.

Luís Martín-Pérez (abril 2013)

Jefe del área Internet de la Cadena Cope.
Maribel Sánchez (julio 2012)

Antigua responsable y creadora del Departamento de Documentación de la Cadena Cope.

Mónica Muñoz-García (noviembre 2012)

Coordinadora de ondacero.es y europafm.com

\section{Bibliografía}

Bueno-Campos, Eduardo (2004). "Fundamentos epistemológicos de dirección del conocimiento organizativo: desarrollo, medición y gestión de intangibles". Economía industrial, n. 357, pp. 13-26.

http://goo.gl/U5Vwid

Carrillo-Durán, María-Victoria; Nuño-Moral, María-Victoria (2010). "La documentación en la evaluación y gestión de la imagen corporativa". El profesional de la información, v. 19, n. 2, pp. 123-132.

http://dx.doi.org/10.3145/epi.2010.mar.02

Cox, Andrew; Yeadon, Jane (2002). "Estrategias prácticas en gestión de contenidos". El profesional de la información, v. 11, n. 6, pp. 466-474.

http://www.elprofesionaldelainformacion.com/contenidos/2002/noviembre/7.pdf

http://dx.doi.org/10.1076/epri.11.6.466.15462

Davenport, Thomas H.; Prusak, Laurence (2001). Conocimiento en acción. Cómo las organizaciones manejan lo que saben. Madrid, Pearson Educación. ISBN: 9789879460290

De-Mateo, Rosario; Bergés, Laura; Sabater, Marta (2009). Gestión de empresas de comunicación. Sevilla: Comunicación Social. ISBN: 9788496082908

Fernández-Marcial, Viviana (2006). "Gestión del conocimiento versus gestión de la información". Investigación bibliotecológica, v. 20, n. 41, pp. 44-62.

http://www.ejournal.unam.mx/ibi/vol20-41/IBI002004102. $p d f$

Fernández-Sande, Manuel; Peinado-Miguel, Fernando (2012). "La empresa radiofónica actual”. En: Gallego-Pérez, Juan-Ignacio; García-Leiva, Trinidad. Sintonizando el futuro: radio y producción sonora en el siglo XXI. Madrid: Instituto RTVE. pp. 29-61. ISBN: 9788488788856

García-Lastra, José-María (2012). “Del modelo productivo de la era analógica al de la radio multicanal". En: GallegoPérez, Juan-Ignacio; García-Leiva, Trinidad. Sintonizando el futuro: radio y producción sonora en el siglo XXI. Madrid: Instituto RTVE. pp. 167-188. ISBN: 9788488788856

García-Morales, Elisa; Bustelo-Ruesta, Carlota (2001). "Tendencias en la gestión de la información, la documentación y el conocimiento en las organizaciones". El profesional de la información, v. 10, n. 12, pp. 4-7.

http://eprints.rclis.org/10040

http://dx.doi.org/10.1076/epri.10.12.4.6540

Newman, Victor (1997). "Redefining knowledge management to deliver competitive advantage". Journal of knowledge management, v. 1, n. 2, pp. 123-128. http://dx.doi.org/10.1108/EUM0000000004587

Nonaka, Ikujiro; Takeuchi, Hirotaka (1995). The knowled- 
ge creating company. Oxford University Press. ISBN: 978 0195092691

Pearson, Thomas A. (1999). "Measurement and the knowledge revolution". Quality progress, v. 32, n. 9, pp. 31-37.

Peinado-Miguel, Fernando; Rodríguez-Barba, Dolores (2011). "Nuevos modelos de negocio para la radio". En: Ortiz-Sobrino, Miguel-Ángel; López-Vidales, Nereida. Radio 3.0 una nueva radio para una nueva era: la democratización de los contenidos. Madrid: Fragua. pp. 141-162. ISBN: 978 8470744365

Pérez-Montoro, Mario (2005). "Sistemas de gestión de contenidos en la gestión del conocimiento". BiD: textos univer- sitaris de biblioteconomia i documentació, n. 14. http://www.ub.edu/bid/14monto2.htm

Robertson, James (2003). "So, what is a content management system". Step two designs, June.

http://www.steptwo.com.au/papers/kmc_what/index.html

Rodríguez-Pallares, Miriam (2012). "El capital intangible como clave estratégica en la empresa radiofónica española: el caso de la cadena Ser". En: Fernández-Sande, Manuel; Adami, Antonio (coord.). Panorama da comunicação e dos meios no Brasil e Espanha / Panorámica de la comunicación y de los medios en Brasil y España. São Paulo (Brasil): Intercom, pp. 473-495. ISBN: 9788582080153

http://www.intercom.org.br/e-book/panorama.pdf
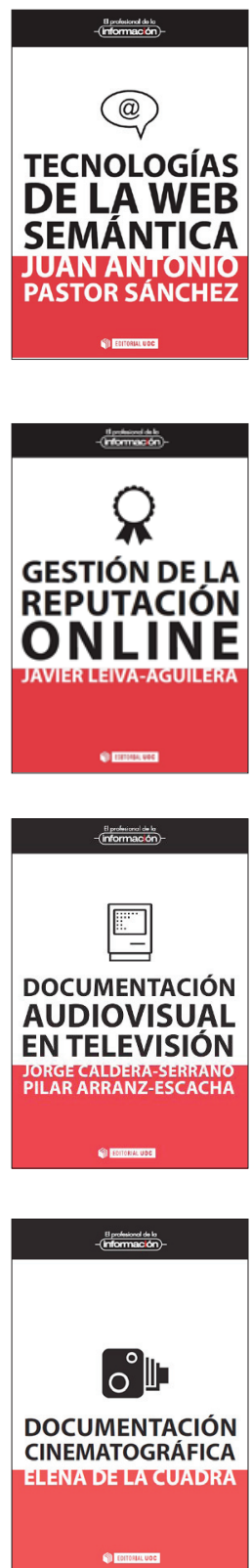
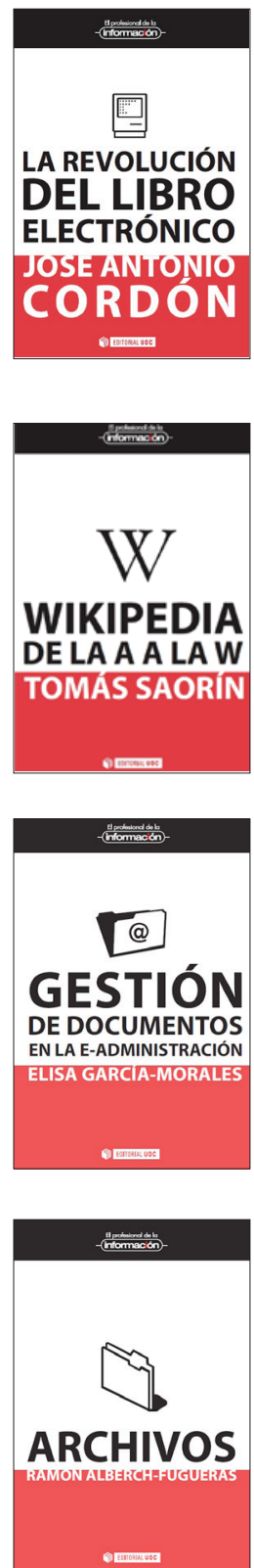
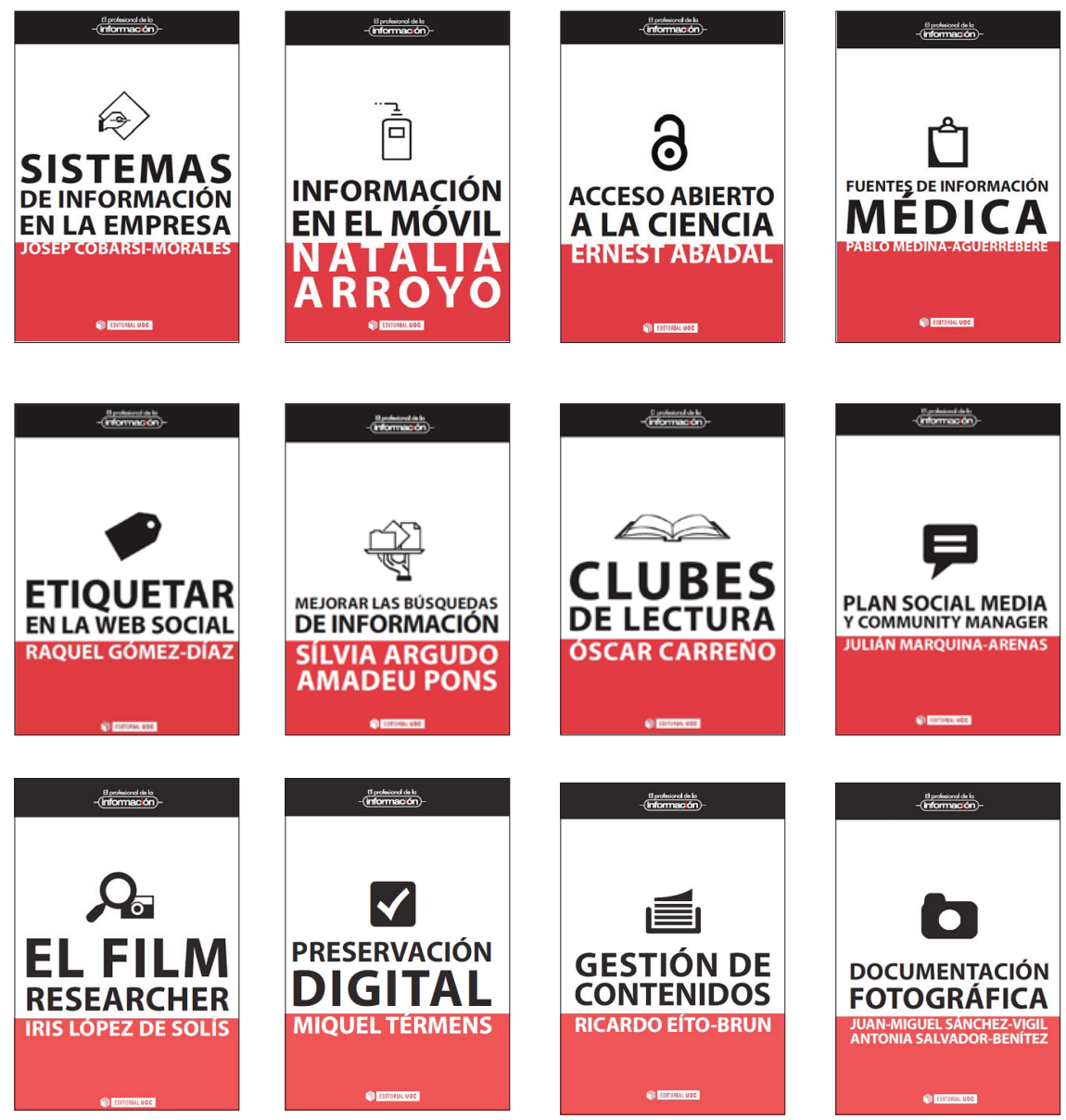

Más información:

http://www.elprofesionaldelainformacion.com/libros.html 